\title{
Power supply requirements planning research in distribution of power
}

\section{grid}

\author{
ZHAO Yu Bo, WANG Ge, CHEN Zhu Xin, LIU Li Zhong and LIU Feng Zhen
}

State Grid Liaoning Chaoyang Power Supply Company, Chaoyang, Liaoning,122000, China

Keywords: power grid; Load; Off-peak; Scheduling

Abstract. Based on a detailed analysis and research of power system load power consumption demand model, on the basis of through offline and online status of demand planning, design a kind of optimal power grid scheduling policy. The scheme can minimize a cycle of the grid operating costs, then strengthen the rationality of the system overall energy distribution and global power supply reliability.

\section{Introduction}

Smart grid is mainly the use of information and communication technologies improve system flexibility and reliability, the rational use of energy, make the system integration of various types of power supply resources, including renewable, distributed generators, storage power station and plug-in electric cars, etc. The rapid development of the smart grid and advanced metering, automation, two-way communication, new technologies, such as distributed generation and storage. Through the Internet address unit real-time interactive communication between the consumers and operators.

Design and implementation of the smart grid with power supply and load demand and efficient management of capital construction goal. Quality of power supply is the system operation, the basic task is to through the peak period of transfer power to the emergency load to reduce peak demand.

Load management does not significantly reduce the total energy consumption demand, as most reduce work load from peak to peak time intervals. However, by avoiding overload operation cycle helps to slow the demand of the whole cycle. Below the critical load of power supply total demand reduce voltage fluctuation caused by the instability, which in turn improve system reliability. More importantly, in addition to the load management to reduce or to meet the needs of the peak power grid electricity generated. This part of the electrical energy based consumer price higher than average, because it is from the micro gas turbine or imported from other countries, and the price. Therefore, from the system running, efficient lowering the operating costs for the power grid load management perspective; From the Angle of users reduce the real-time electricity price.

In this article, first of all, to develop and solve the problem of basic control and optimization of power grid operation. Addressing the intelligent metering device through the network real-time communication between operators and consumers. Grid operators complete control of consumer appliances, operating controller receives power requests from different customers. Each request have different power requirements, time, and elastic. Elastic section of the planning for each load demand deadline. Grid operators is the purpose of designing a power demand task scheduling strategy, minimize a time scale power grid operating costs. Total power consumption of the 
instantaneous operating costs is modeled as a system function, and with the increase of total power demand, for each additional unit of electricity demand higher cost.

\section{System Modeling}

Assuming all tasks can eventually planning demand, for their deadline, the latest demand task no loss in the system. A task planning to happen, or after if, in the aftermath of the activated duration until he finished the task. Therefore, each task arrangement in time, or when the continuation of task arrival time:. To plan in advance, can only be done on time.

Controller receives the power demand from intelligent device activation time is determined. Then issue the corresponding activation command to the smart devices, and then passed to the corresponding electric appliances, make it work. Period of manipulation of the controller to activate power requirements as shown in figure 2. Controller and intelligent equipment and user electrical communication via network connection so as to realize zero delay at a high speed.

From controller minimum running cost, if the power consumption demand time, duration and deadlines, known for an offline planning is feasible, and the boundary conditions can be obtained. Starting from the long-term average cost minimization, for random power requirements are available online analysis method.

For each time $t, p(t)$ as the instantaneous power consumption of the system. $T$ point of instantaneous power consumption is defined as $\mathrm{C}(\mathrm{P}(\mathrm{t}))$, it can be derived function, to increase and the function of reactive power controller difference power cost increases with an increase in demand. Each more to meet user demand for power unit costs are higher.

\section{Offline requirements planning}

First of all, the cognizance of offline programming tasks for the $\mathrm{n}$ power demand. Task respectively, $\mathrm{n}=1,2 \ldots \mathrm{N}$, starting time, power consumption, duration, and the deadline. Controller in the early time to determine the amount, if the parameter bounds, is the whole problem can be expressed.

To advance scheduling tasks: first, considering the elastic demand case, every job tasks can be accordingly in advance, and no corresponding necessary in a row. Each task can be interrupted, then continue, both in the continuous time get incentives, the task can be completed before at a certain time, after every job is to motivate all have fixed power requirements.

Each task $\mathrm{n}$ and time $\mathrm{t}$, a function, if a task $\mathrm{n}$ at time $\mathrm{t}$, motivated, planning task can be said function. Defined time interval [0, T]. Planning strategy for the controller is within the scope of [0, T] minimize the total cost. For each time t, instantaneous loss function for the total instantaneous power load. Controller of the optimization problem is:

\section{Constraints:}

Suitable for $\mathrm{n}=1,2 \ldots, \mathrm{N}$ and. Means that every job had to be done within their respective deadlines. 
For scheduling tasks in advance: as inelastic demand. Once scheduled start, work will be continued until you're done. Discrete time analysis is more suitable for capturing the situation. For each task $\mathrm{n}=1,2 \ldots, \mathrm{N}$, and deadlines at the beginning, at the same time assume their electricity demand is the same, that is. Find a positive integer, and consider whether or not the maximum instantaneous power $\mathrm{N}$ task for.

The duration of each task is defined as, defined as $\mathrm{D}$ cycle range. The above problem is equivalent to one dimensional space, whether for a series of $\mathrm{N}$ task set can be divided into $\mathrm{m}$ a disjoint subsets, thus the size of each subset is D, or even smaller. Obviously each subset is a one-dimensional function of the instantaneous power $\mathrm{p}, \mathrm{m}$ a subset is enough to meet $\mathrm{N}$ task, thus the maximum instantaneous power consumption is.

\section{Online dynamic demand planning}

The needs of online dynamic programming problem continuously produce and to make planning decisions as the system running. Transmitted to the grid operator's power requirements in line with the poisson process, lambda said average instantaneous rate per unit time. Each time the duration of the power demand as random variables, as is described by using exponential form, at an average rate of $\mathrm{S}$ requirements for the power of time, the duration of the different needs of independent random variables.

Request for each $\mathrm{n}=1,2 \ldots, \mathrm{N}$ deadline exponentially distributed, the same as, $\mathrm{d}$ for deadlines, at an average rate of deadline is also independent of different request, minimum run average cost is:

Mathematical expectation about the stationary distribution of $\mathrm{P}(\mathrm{t})$, the controller can measure the total instantaneous power consumption, so as to set up the system state of reliable control strategy model.

\section{Conclusion}

In this paper, a preliminary established the smart grid control and optimization theory. The fundamental problem of the power grid is studied for peak power demand, minimizing the scope for a period of time the grid operating costs, effectively promote energy management. Analysis of offline programming problem, the optimal solution from the allow dispatching the elasticity of demand in advance; And for the scheduling in advance can be classified into polynomial inelastic demand. In view of the dynamic programming system requires online decision-making, run by stochastic model to establish a long-term expected costs. Main research results is the instantaneous power consumption are given, with the optimal control strategy to minimize power consumption costs have been gradually.

\section{References}

[1] shu-yong Chen, Song Shufang Li Lanxin etc. The smart grid technology review [J]. Power grid technology, 2009 (8) : 1-7. 
[2] Lin, $\mathrm{m}$ for the people, titiyeah etc. Some problems in intelligent scheduling modeling technology research [J]. Power grid technology, 2011, 35 (6) 1-4.

[3] k. Moslehi and r. Kumar, "Smart Grid: A Reliability Perspective", Proc. IEEE PES Conference on Innovative Smart Grid Technologies, 2010.

[4] Du Guihe Wang Zhengfeng. Smart grid scheduling integration design and research [J]. Power system protection and control. 15 issue of 2010.

[5] Senjyu, y. t. Miyazato, a. Yona, N.U rasaki, and t. Funabashi, "Optimal distribution voltage control and the coordination with distributed generation," Power Delivery, IEEE the Transactions on, vol. 23, no. 2, pp. 1236-1242, 2008.

[6] g. Xiong, c. Chen, s. Kishore, and a. Yener, "Smart power scheduling for demand response on the Smart grid," in proc. Innovative Smart grid Technologies, 2011.

[7] Pan Yi Zhou Jing Yang, li qiang, etc. The power system model based on common information model of the split and merge [J]. Automation of electric power systems, 2003, 27 (15) : 45-48.

[8] jian-guo yao village, sheng-chun Yang etc. Key techniques of intelligent dispatching [Cl. 2009 international conference on uhv transmission technology, Beijing, 2009. 